\title{
Chemical Doping of Graphene by Altretamine(2,4,6-Tris [dimethylamino]-1,3,5-Triazine)
}

\author{
Sunmin Park, Sena Yang, Heeseon Lim, and Hangil Lee* \\ Department of Chemistry, Sookmyung Women's University, Seoul 140-742, Korea. *E-mail: smj0090@sookmyung.ac.kr \\ Received March 29, 2011, Accepted May 11, 2011
}

\begin{abstract}
The electronic properties of altretamine(2,4,6-tris [dimethylamino]-1,3,5-triazine) adsorbed on epitaxial graphene (EG) were investigated by core-level photoemission spectroscopy (CLPES) in conjunction with low energy electron diffraction (LEED). We found that altretamine molecule adsorbed onto interface layer (S1) of graphene as we confirm decrement of S1 peak using CLPES and haziness of LEED pattern. Moreover, the measured work function changes verified that increased adsorption of the altretamine on graphene layer showed $n$-type doping characteristics due to charge transfer from altretamine to graphene through the nitrogens. Two distinct nitrogen bonding feature associated with the $\mathrm{N} 1 s$ peak was clearly observed in the core-level spectra indicating two different chemical environments.
\end{abstract}

Key Words : Altretamine, Chemical doping, Epitaxial graphene, CLPES, LEED

\section{Introduction}

Graphene, which is formed by a single layer of graphite with two-dimensional $s p^{2}$ orbital, has recently attracted significant attention due to its exceptional electronic and optical properties. ${ }^{1}$ Its varied applications in many emerging areas, such as graphene-based electronic devices, have motivated many research groups to actively study the material's properties. It may be possible to handle both the occupation of electronic states near the Fermi level and the magnitude of the gap between the valence band and the conduction band by controlling the carrier density using metals or molecules in the graphene layer. ${ }^{2-7}$

Chemical functionalized molecular adsorption on inorganic surfaces is a indispensable step in processes of great scientific and technological importance, such as crystallization and heterogeneous catalysis. ${ }^{8}$ Successful engineering of thin molecular films requires careful analysis of many factors, including the structure of the individual components, their interactions with one another, and their affinity for the underlying surface. For adsorption on atomically flat surfaces, molecules that can adopt planar topologies are ideal, and their 2D organization can often be controlled predictably when they engage in strong directional intermolecular interactions such as hydrogen bonds. ${ }^{9}$ As a point of view, graphene is a very fascinating surface due to the flatness and inertness.

In this study, we doped monolayer graphene with altretamine (2,4,6-tris [dimethylamino]-1,3,5-triazine: altretamine). Altretamine is a synthetic, cytotoxic, antineoplastic, $s$ triazine derivative that can be taken verbally. The exact mechanism of action is unknown, although chemically it resembles alkylating agents. ${ }^{10,11}$ The energies and geometries of the adsorption of altretamine on graphene are directly related to the ability of the molecules to accommodate additional electron density arising from charge transfer contributed by the graphene sheet. Charge transfer from graphene to aminotriazines occurs in part through the presence of hydrogen atoms in the substituents, which explains why terminal hydrogen atoms are directed toward graphene surface. Related variations in the magnitude of charge transfer as a function of the structure of bound molecules may have useful applications, such as in devising non-invasive methods for hole-doping carbon-based nanomaterials. ${ }^{12}$

The ability of melamine to bind much more strongly and accept more charge reflects the higher electronegativity of nitrogen, which decreases the net charge on the hydrogen atoms of the $\mathrm{NH}_{2}$ groups and thereby allows them to accept additional density contributed by graphene. This effect is magnified by $\mathrm{NMe}_{2}$ substituents, which provide a larger number of hydrogen atoms for interaction with the surface. In addition, these hydrogen atoms can be directed toward the surface without requiring substantial pyramidalization of nitrogen atoms. Truly, the sum of the bond angles around nitrogen in the $\mathrm{NH}_{2}$ groups of melamine is only $334.9^{\circ}$, whereas that in the $\mathrm{NMe}_{2}$ groups of altretamine $\left(359.9^{\circ}\right)$ is close to the ideal $s p^{2}$ value (nearly $\left.360^{\circ}\right){ }^{13}$

Hence, we predicted that altretamine could successfully functionalize the graphene surfaces because it includes $\mathrm{NMe}_{2}$ groups that may be used to facilitate chemical doping. Increasing the deposition of altretamine was expected to produce doping and to modulate EG properties. The studied graphene have been characterized by core-level photoemission spectroscopy (CLPES) in conjunction with Low energy electron diffraction (LEED).

\section{Experimental Methods}

CLPES measurements were performed under ultrahigh vacuum conditions (base pressure $=1.5 \times 10^{-10}$ Torr) at the 8A2 beamline of the PAL (Pohang Accelerator Laboratory) in pohang, which is equipped with an electron analyzer 


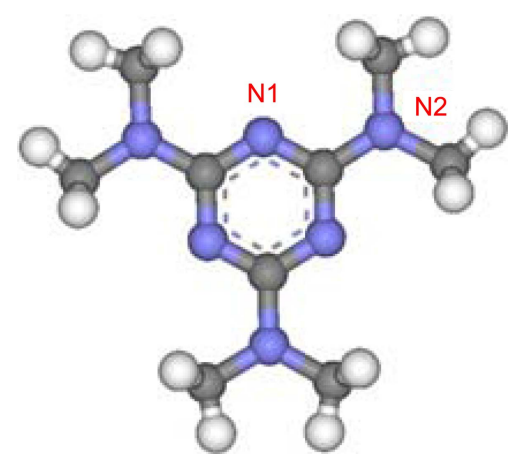

Figure 1. Structure of altretamine(2,4,6-tris [dimethylamino]1,3,5-triazine) molecule. Each blue, gray and white ball indicates nitrogen, carbon and hydrogen, respectively.

(SES100, Gamma Data Scienta). The annealing temperature was monitored with an infrared pyrometer assuming an emissivity of 0.90 . The nitrogen-doped $\left(\mathrm{N}_{\mathrm{D}} \sim 9 \times 10^{17} \mathrm{~cm}^{-3}\right)$ Si-terminated $6 \mathrm{H}-\mathrm{SiC}(0001)$ substrate was purchased from Cree Research (USA). After degassing the substrate to 900 ${ }^{\circ} \mathrm{C}$ for 24 hours, we deposited silicon $(1 \AA / \mathrm{min})$ for 5 minutes at $900{ }^{\circ} \mathrm{C}$ to produce a Si-rich surface. Finally, the substrate was graphitized at temperatures up $1150-1200{ }^{\circ} \mathrm{C}$ for two minute to produce monolayer graphene. The thickness of graphene can be controlled by the graphitization temperature and time. Altretamine (Sigma Aldrich, purity $-96 \%$ : see Figure 1) was purified by turbo pumping to remove impurities before dosing on the EG surface. A direct solid doser was used to introduce the altretamine. The $\mathrm{C} 1 \mathrm{~s}$ and $\mathrm{N} 1 s$ core-level spectra were obtained using photon energies of 320 and $510 \mathrm{eV}$. Secondary electron emission spectra $(-20 \mathrm{~V}$ sample bias) were measured at a photon energy of $80 \mathrm{eV}$. The binding energies of the core-level spectra were determined with respect to the binding energies of the clean $\mathrm{Au} 4 f$ core level and the valence band (Fermi energy) for the same photon energy. All spectra were recorded in the normal emission mode. The photoemission spectra were carefully analyzed using a standard nonlinear least-squares fitting procedure with Voigt functions. ${ }^{14}$

\section{Results and Discussion}

In order to describe the structure of altretamine adsorbed onto EG, the CLPES measurements were performed and results are presented in Figure 2. A monolayer graphene grown on $6 \mathrm{H}-\mathrm{SiC}(0001)$ obtained after annealing at $1180{ }^{\circ} \mathrm{C}$ for 2 minute. Figure 2(a) shows the $\mathrm{C} 1 s$ core-level spectrum of the graphene layer which thickness was 1.0 ML (monolayer) at this annealing temperature, as confirmed by CLPES (attenuation of the Si $2 p$ spectra, data not shown) and lowenergy electron diffraction (LEED) image shown in Figure 4(a). ${ }^{15}$ As shown in Figure 1(a), we found four distinct peaks emerged at 285.1, 285.6, 284.7, and $283.6 \mathrm{eV}$, which were assigned, respectively, $(6 \sqrt{3} \times 6 \sqrt{ } 3) R 30^{\circ}$ interface layer (buffer layer marked S1), to $s p^{2}$ carbon atoms in the interface layer weakly interacting with the carbon atoms of the underlying substrate (marked S2), graphene layer (marked as $\mathrm{G}$ ), and $\mathrm{SiC}$ (marked as $\mathrm{SiC}$ ). ${ }^{16,17}$ After confirming the pristine state of monolayer graphene by $\mathrm{C} 1 \mathrm{~s}$ core-level spectrum, we exposed EG to 24 and $336 \mathrm{~L}$ altretamine.
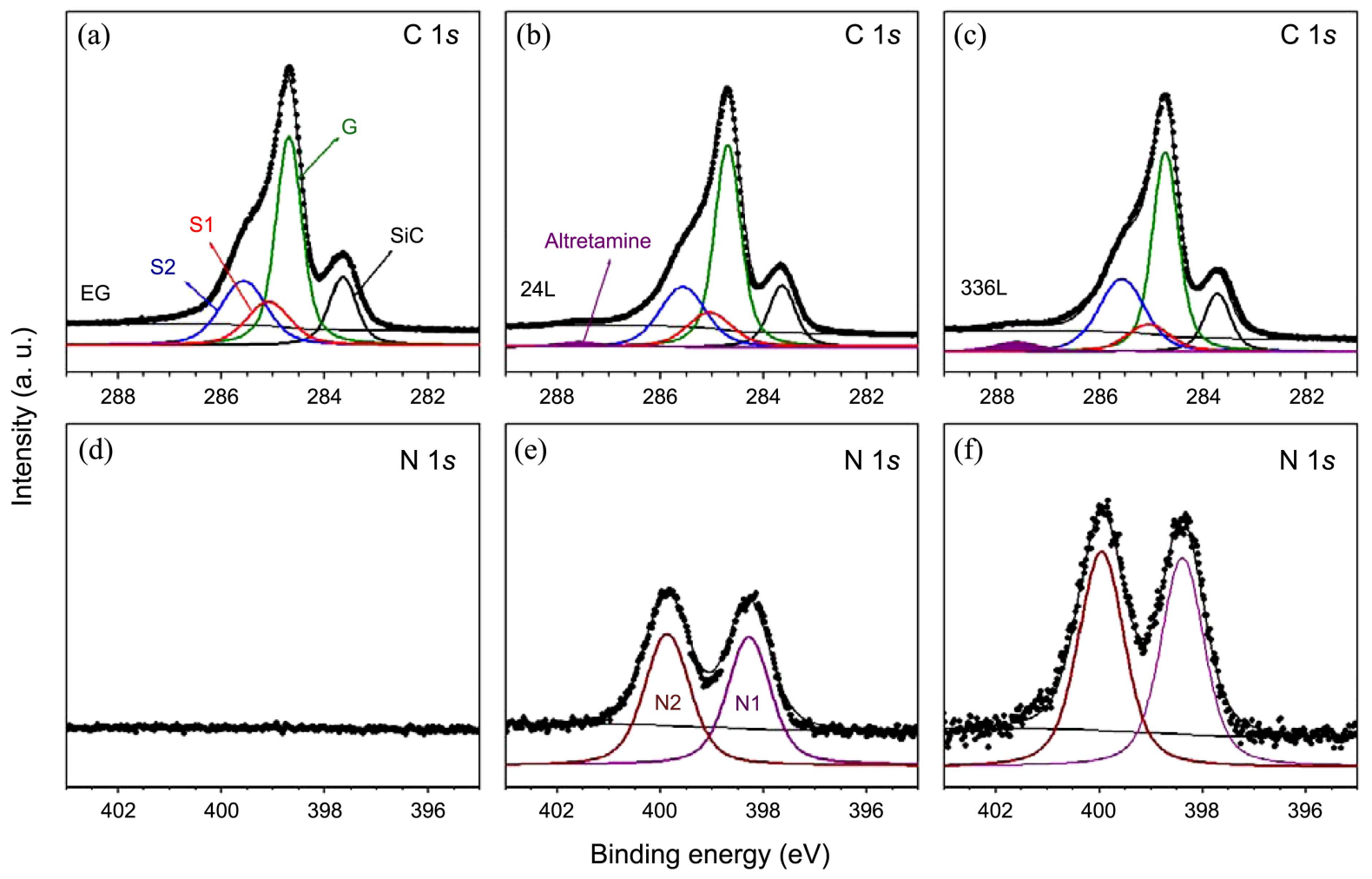

Figure 2. (a-c) C $1 s$ core-level spectra obtained at photon energy of $320 \mathrm{eV}$. (d-f) N $1 s$ core-level spectra obtained at photon energy of 510 eV. (a) and (d) $1.0 \mathrm{ML}$ of graphene, (b) and (e) altretamine deposition $24 \mathrm{~L}$ at $100{ }^{\circ} \mathrm{C}$, (c) and (f) $336 \mathrm{~L} \mathrm{at} 100{ }^{\circ} \mathrm{C}$. 
Figure 2(b) and (c) show the C $1 s$ core-level spectra after the adsorption of altretamine (24 L and $336 \mathrm{~L}$ ) on graphene, during which the substrate temperature was maintained at $100{ }^{\circ} \mathrm{C}$ to enhance the adsorption rate. In these spectra, we observed a decrease in the intensity of the $\mathrm{S} 1$ peak about $15 \%$ at $24 \mathrm{~L}$ and about $28 \%$ at $336 \mathrm{~L}$ altretamine induced by the interfacial carbon. Because S1 included several defects, a decrease in this peak intensity due to altretamine exposure was reasonable. Moreover, we obtained a new peak (marked Altretamine) increase in the intensity by the adsorption of altretamine onto EG.

Interestingly, although a sufficient altretamine is adsorbed on EG, we do not observe any change of graphene peak such as binding energy, intensity as well as shape. It is clearly noted that the adsorbed altretamine does not affect the morphology of graphene layer. As a result, we can clearly demonstrate that excluding two phenomena, we found no changes in the $\mathrm{C} 1 s$ spectrum and we can expect that the occurred interaction between altretamine and graphene is $\pi-\pi$ interaction or van der Waals interaction, respectively.

Next, we acquired N $1 s$ core-level spectra to chase the adsorption of altretamine onto EG. Our focus was to confirm the presence and bonding features of the nitrogen atoms in the altretamine. The N $1 s$ core-level spectrum of the system with altretamine adsorbed on graphene (Fig. 1(e) and (f)) displayed two distinct peaks located at 398.3 (marked N1) and $399.9 \mathrm{eV}$ (marked N2), which were assigned to $-\mathrm{C}=\mathrm{N}$ and $\mathrm{NR}_{2}$. The part of the driving force for adsorption is a specific attractive interaction of $\mathrm{NR}_{2}$ groups with the underlying graphene surface. Hence, through spectral analysis, we obviously can explain that altretamine adsorbed onto EG without bond breakage. As discussed above, the EG surface includes an interface layer. Our substrate showed metallic behavior due to the formation of an additional graphene layer on the interface layer. From the CLPES result, we did not observe the variation of graphene peak and $\mathrm{N} 1 s$ peaks being included in altretamine molecule although we increased its exposing up to $336 \mathrm{~L}$. It can be clearly explained that altretamine only adsorb onto interface layer without any change of structures.

We also measured the work function as a function of altretamine exposure by monitoring the low kinetic energy cutoff region, as shown in Figure 3 to confirm the altretamine induced doping effect. Global doping effects on EG were assessed by measuring the difference of the secondary electron edge between what two systems, which is equal to the work function difference. ${ }^{18}$ The black spectrum displayed in Figure 3(a) corresponds to pristine monolayer graphene reference spectrum. As we increased the coverage of altretamine up to $336 \mathrm{~L}$, we clearly observed that the secondary electron edge shifted to lower kinetic energies by $340 \mathrm{meV}$ with respect to pristine monolayer graphene. In other words, molecular doping by altretamine decreased the work function of graphene indicating a typical $n$-type doping. The inset shown in Figure 3 displays the decrease in the work function of monolayer graphene, up to $3.96 \mathrm{eV}$, with increased doping of altretamine. This shift indicated $n$ -

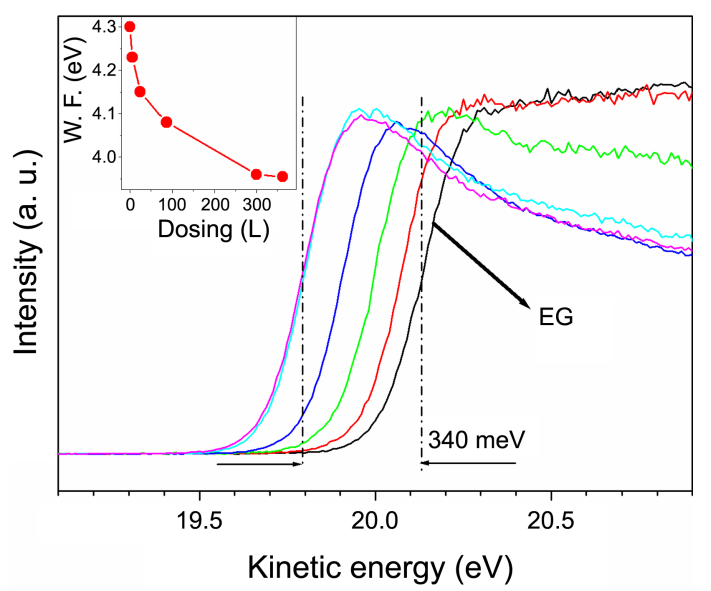

Figure 3. Work function measurements using the secondary electron cutoff with photon energy of $80 \mathrm{eV}$ and a sample bias of -20 V. Pristine monolayer graphene, $24 \mathrm{~L}$ and $336 \mathrm{~L}$ altretamine adsorbed on EG. The inset shows the decrease in the work function as a function of altretamine exposure.

type doping. We assumed that the work function of monolayer graphene was $4.30 \mathrm{eV} .^{19}$ The increment in the work function results from the presence of a dipole on graphene, produced by the adsorption of altretamine with $\mathrm{NMe}_{2}$ groups, which provide a larger number of hydrogen atoms for interaction with the surface. Similar phenomena have been found in thiol/Au systems, in which the lowering of the work function was also observed. ${ }^{20}$ The electronic structure of EG has been controlled previously using gold and $p$-tertbutylcalix[4]arene ( $p$-type doping).,21

Figure 4 shows an evolution of LEED patterns observed upon altretamine adsorption. The initial surface, monolayer graphene, reveals a distinct $(6 \sqrt{3} \times 6 \sqrt{3}) \mathrm{R} 30^{\circ}$ interface related pattern (red circle) in Figure 4(a). After altretamine adsorption, a blurry $(6 \sqrt{3} \times 6 \sqrt{3})$ R $30^{\circ}$ LEED pattern emerges in Figure 4(b). Those LEED spots upon altretamine adsorption become blurred and explain evidence of adsorption on interface region. It is noticeable that the change of the LEED pattern from Figure 4(a) to Figure 4(b) with altretamine adsorption seems to be related to adsorption on interface region, as shown by the intensity change of the $\mathrm{S} 1$ peak in Figure 2.

Figure 5 displays the schematic diagram of the expected adsorption geometry of altretamine adsorbed on EG. This model is characterized by the presence of interactions bet-
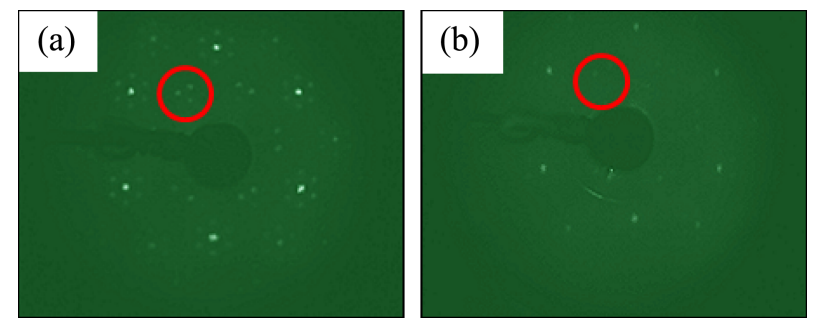

Figure 4. (a) LEED images of EG grown on on $6 \mathrm{H}-\mathrm{SiC}(0001)$ at $1180{ }^{\circ} \mathrm{C}$ for 2 minute and (b) LEED image obtained after exposing of $336 \mathrm{~L}$ altretamine on EG. 

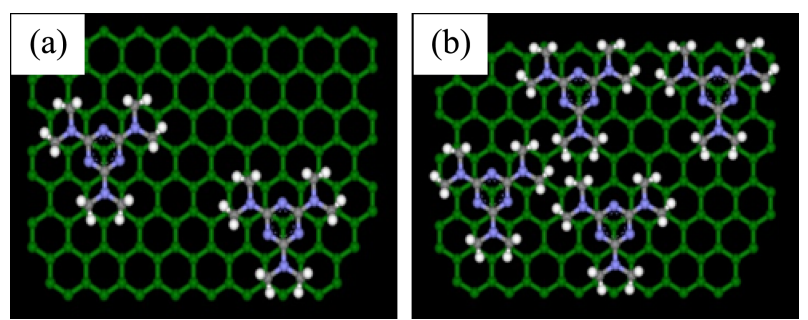

Figure 5. Schematic diagrams for altretamine adsorbed on EG (top view). Each blue, gray, white, and dark cyan ball indicates nitrogen, carbon, hydrogen, and graphene, respectively.

ween the $\pi$-electron densities of graphene and the triazine ring of altretamine. The molecules are more-or-less parallel to each other and are displaced in such a manner that one of the nitrogen atoms of the triazine ring is centered on the graphene and the substituted carbon atom of graphene is centered over the triazine ring. As shown in Figure 5, the altretamine are expected to adsorb on the top sites of EG to form $\pi-\pi$ interaction or vander waals interaction.

\section{Conclusions}

In conclusion, we observed that the chemical doping of EG with altretamine is characterized by $n$-type doping. By using CLPES, we confirmed the lowering of the work function (n-type) and the variations in the electronic structure of adsorbed on EG with increasing doses of altretamine. Additionally, LEED measurements noted that altretamine molecule adsorb on interface layer (marked S1 in Figure 2). We believe that the chemical functionalized molecular doped graphene layer may possibly be utilized as an active center for the chemical functionalization of EG and be useful for various applications.

Acknowledgments. This work was supported by the Sookmyung Women's University Research Grants 2010.

\section{References}

1. Geim, A.; Novoselov, K. Nature Mater. 2007, 6, 183.

2. Ohta, T.; Bostwick, A.; Seyller, T.; Horn, K.; Rotenberg, E. Science 2006, 313, 951 .

3. Gierz, I.; Riedl, C.; Starke, U.; Ast, C. R.; Kern, K. Nano. Lett. 2008, 8,4603 .

4. Bekyarova, E.; Itkis, M. E.; Ramesh, P.; Berger, C.; Sprinkle, M.; de Heer, W. A.; Haddon, R. C. J. Am. Chem. Soc. 2009, 131, 1336.

5. Zhou, S. Y.; Siegel, D. A.; Fedorov, A. V.; Lanzara, A. Phys. Rev. Lett. 2008, 101, 086402.

6. Chen, W.; Chen, S.; Qi, D. C.; Gao, X. Y.; Wee, A. T. S. J. Am. Chem. Soc. 2007, 129, 10418.

7. Liu, L. H.; Yan, M. Nano Lett. 2009, 9, 3375.

8. Elemans, J. A. A. W.; Lei, S.; De Feyter, S. Angew. Chem., Int. Ed. 2009, 48, 7298.

9. Zhou, H.; Dang, H.; Yi, J.-H.; Nanci, A.; Rochefort, A.; Wuest, J. D. J. Am. Chem. Soc. 2007, 129, 13774.

10. Ames, M. M. Cancer. Treat. Rev. 1991, 18, 3.

11. Rhoda Lee, C.; Faulds, D. Drugs 1995, 49, 932.

12. Biel, B.; Triozon, F.; Blasé, X.; Roche, S. Nano Lett. 2009, 9, 2725.

13. Wuest, J. D.; Rochefort, A. Chem. Commun. 2010, 46, 2923.

14. Schreier, F. J. Quant. Spectrosc. Radiat. Transfer. 1992, 48, 743.

15. Kim, K.-J.; Lee, H.; Choi, J.; Lee, H.-K.; Kang, T.-H.; Kim, B.; Kim, S. J. Phys.: Condens. Matter. 2008, 20, 225017.

16. Emtsev, K. V.; Bostwick, A.; Horn, K.; Jobst, J.; Kellogg, G. L.; Ley, L.; McChesney, J. L.; Ohta, T.; Reshanov, S. A.; Röhrl, J.; Rotenberg, E.; Schmid, A. K.; Waldmann, D.; Weber, H. B.; Seyller, T. Nature Mater. 2009, 8, 203.

17. Holzinger, M.; Abraham, J.; Whelan, P.; Graupner, R.; Ley, L.; Hennrich, F.; Kappes, M.; Hirsch, A. J. Am. Chem. Soc. 2003, $125,8566$.

18. Cahen, D.; Kahn, A. Adv. Mater. 2003, 15, 271.

19. Alloway, D. M.; Hofmann, M.; Smith, D. L.; Gruhn, N. E.; Graham, A. L.; Colorado, R., Jr.; Wysocki, V. H.; Lee, T. R.; Lee, P. A.; Armstrong, N. R. J. Phys. Chem. B 2003, 107, 11690.

20. Hibino, H.; Kageshima, H.; Kotsugi, M.; Maeda, F.; Guo, F.-Z.; Watanabe, Y. Phys. Rev. B 2009, 79, 125437.

21. Park, S.; Yang, S.; Kim, K.-J.; No, K.-H.; Lee, H. Bull. Korean Chem. Soc. 2010, 31, 2809. 